En juillet 1994, le CNRS a créé un Comité d'éthique pour les sciences. Cela traduit avec force une sensibilité institutionnelle nouvelle concernant la recherche et le métier de chercheur, ainsi que la place et le rôle des sciences et des scientifiques dans la société ! Ce Comité a maintenant deux années d'expérience, suffisamment pour commencer à donner corps à un terme qui demeure aussi vague que nécessaire. Il a paru intéressant de faire le point avec les principaux protagonistes de cette initiative et de présenter un dossier sur ce Comité.

\title{
ENTRETIEN AVEC FRANÇOIS KOURILSKY
}

352

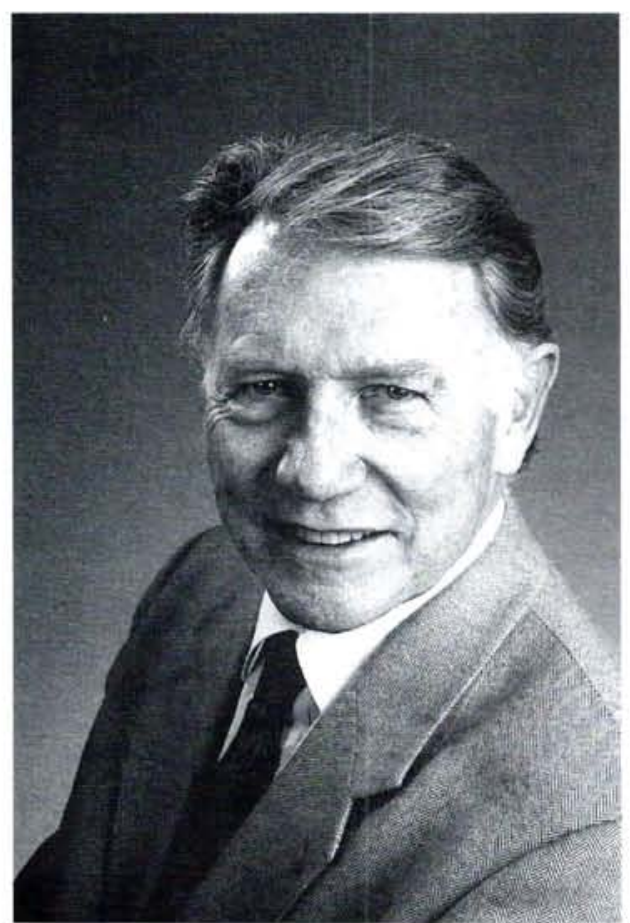

François Kourilsky (voir notice p. 357)

* Ce dossier donne une image de la situation du COMETS au moment des entretiens (1995), soit un an apres sa crèation. Nous ferons le point sur ses evolutions courant 1997.
NSS — Comment l'idée d'un Comité d'éthique pour les sciences est-elle née ? Comment a-telle fini par s'imposer au sein d'un organisme comme le CNRS?

En cette fin de siècle, la croyance dans une science " source de bienfaits pour l'humanité " s'est fortement affaiblie. La menace nucléaire, la conférence de Rio sur l'environnement global, les craintes des manipulations génétiques sont passées par là. C'est la formidable accélération des nouvelles technologies issues de la science, la prise de conscience par les scientifiques des effets pervers de certaines utilisations des progrès scientifiques qui ont amené le besoin d'une éthique des sciences et l'ont finalement imposée. L'attitude consistant à désolidariser la technologie (source de risques) de la science (restée innocente et pure) n'est pas plus fondée qu'elle n'est acceptable. Le problème ne date pas d'aujourd'hui. Les états d'âme des scientifiques mêlés à la fabrication de la bombe atomique en sont assurément l'exemple le plus célèbre. Mais il n'est pas le seul ! Dans le domaine médical, le moratoire d'Asilomar sur les manipulations génétiques, les prises de positions de Jacques Testart sur la procréatique, les débats sur les essais thérapeutiques..., furent des symptômes moteurs.

NSS = L'affaire du sang contaminé a-t-elle joué un grand rôle?

Non, elle fut postérieure.
NSS - Peut-on dire que c'est dans le domaine médical que le besoin d'un Comité d'éthique s'est fait sentir en premier lieu?

Indubitablement oui. Les interrogations sociales y étaient les plus vives face aux progrès de la biologie. Cela s'est traduit par la création d'un Comité consultatif national d'éthique pour les sciences de la vie et de la santé. Dès les années quatre-vingt, étant alors vice-président du Conseil supérieur de la Recherche et de la Technologie, nous avions débattu, avec Jean Bernard, président du Comité national d'éthique, de l'opportunité d'élargir la réflexion éthique des sciences au-delà de la sphère médicale. En 1989, le projet de création au CNRS d'un groupe de travail sur l'éthique des sciences faisait déjà l'objet de discussions très sérieuses avec nos collègues, physiciens ou philosophes. Le terme "éthique" était introduit en 1991 dans la liste des thèmes d'une section du Comité national de la recherche scientifique. Un colloque interdisciplinaire fut organisé en 1993 au CNRS par Hourya Sinaceur sur "la Recherche éthique et les sciences", afin de recenser les intérêts et travaux scientifiques sur ce thème. Un autre suivit sur les problèmes éthiques de l'entraînement sportif autour de Robert Naquet et Roger Bambuck.

Plusieurs réunions eurent lieu au CNRS en 1993-1994 pour la mise au point du Comité d'éthique pour les sciences. Celuici fut mis en place en 1994 et officialisé peu avant mon départ du CNRS. Comme vous le voyez, tracer un historique simple de cette démarche n'est pas aisé... 
NSS - Avez-vous rencontré des difficultés pour la mise en place du Comité d'éthique pour les sciences du CNRS, difficultés qui on retardé sa création?

Le concept était neuf, l'approche extrêmement interdisciplinaire, et le dialogue au centre de la démarche. Chaque fois qu'il est question d'éthique, et même si le sujet sort brûlant de l'actualité, il est toujours nécessaire de prendre du temps : celui de l'écoute, celui de la réflexion ; le temps du dialogue puis celui du consensus... De plus, le nouveau comité devait être bien inséré dans le paysage institutionnel existant, ce qui n'était pas simple.

NSS Comment situer le Comité d'éthique pour les sciences entre le Comité national d'éthique pour les sciences de la vie et le Comité opérationnel d'éthique du département des sciences de la vie du CNRS?

Le Comité national d'éthique pour les sciences de la vie, placé auprès du Président de la République, est la référence nationale en la matière. Son champ d'intervention n'implique pas tous les domaines scientifiques. Nous avons conçu le Comité d'Éthique pour les Sciences du CNRS comme le complément permettant d'élargir les réflexions au-delà du domaine proprement médical. Le Comité du CNRS enrichira le Comité national. C'est un groupe de réflexion, un espace de dialogue, de confrontation, d'élaboration de consensus, ouvrant le débat éthique au-delà du seul domaine des sciences de la vie. Il est susceptible d'initier des débats, de faire des recommandations au directeur général du CNRS, aux scienti- fiques et de rendre ses avis publics. Or, une recommandation publique s'adresse à qui la lit !

Le COPE (Comité opérationnel d'éthique placé auprès du département des sciences de la vie du CNRS), présidé par Robert Naquet, a été créé pour donner concrètement, dans le cadre du CNRS, des autorisations et recommandations sur des recherches et leur financement ou sur des procédures expérimentales posant des problèmes d'éthique dans le domaine des sciences de la vie. Il ne s'agit donc pas d'un comité de réflexion et son action se limite aux sciences de la vie.

NSS - Pouvez-vous nous donner des exemples de problèmes qui pourraient être, selon vous, du ressort du Comité d'éthique pour les sciences?

Le champ est immense et les problèmes innombrables. Toutefois, à mon avis, seuls méritent d'être abordés au départ par le Comité les sujets concrets, qui sont soulevés par des scientifiques avec une certaine urgence, et qui préoccupent les citoyens. En effet, la réflexion doit motiver fortement les membres du Comité et aboutir à des recommandations concrètes sans trop risquer de s'enliser. Sans être philosophes ou mathématiciens, nous adorons souvent inventer des problèmes théoriques fondés sur des situations supposées, pour le grand plaisir de les résoudre ou d'en discuter à perte de vue, ou à perte d'envie! Les sujets issus de nos réflexions préalables restent donc à trier par la présidente en fonction de l'actualité et des opportunités. Citons quatre exemples : 


\section{COMITÉ D'ÉTHIQUE POUR LES SCIENCES - CNRS}

Le Centre national de la recherche scientifique crée un Comité d'éthique pour les sciences destiné à étendre les réflexions sur les problèmes éthiques des sciences, au-delà des domaines des sciences de la vie et de la médecine.

En effet, la réflexion éthique sur les problèmes posés par l'avancée des connaissances dans le domaine touchant à la médecine et à la biologie, a été largement prise en compte dans notre pays et, en particulier, avec la mise en place du Comité consultatif national pour l'éthique (CCNE) dans le domaine des sciences de la vie et de la santé, en 1984. Des comités opérationnels ont été mis en place dans ce domaine, tels les « comités de protection des personnes dans la recherche biomédicale " en 1991 et, au sein même du CNRS, le Comité opérationnel pour l'éthique dans les sciences de la vie (COPE), en 1991.

Cette réflexion, dans le domaine des sciences de la vie et de la santé, est loin de couvrir l'ensemble du champ de réflexion sur l'éthique des sciences où se posent des problèmes parfois difficiles et méritant une réflexion approfondie. C'est le cas des problèmes relatifs à l'environnement, aux équilibres de la faune et de la flore, aux altérations de l'eau ou des constituants de notre atmosphère. C'est le cas aussi des recherches touchant aux comportements, qu'il s'agisse de psychologie cognitive ou de psychologie sociale. Les sciences de la communication peuvent être aussi à l'origine de pratiques d'influence et de manipulation amplifiées par les technologies modernes de recherches en histoire touchent aussi bien son enseignement, que des manipulations à des fins politiques. Les problèmes posés par les recherches sur l'expérimentation animale, sur l'évolution du nucléaire, fournissent des exemples parmi bien d'autres... Enfin, les problèmes éthiques posés par la transmission du savoir et par la déontologie de la recherche scientifique méritent réflexion. Le CNRS, principal opérateur de la recherche fondamentale en France, de dimension internationale et coul'audiovisuel. Les problèmes engendrés par les

vrant l'ensemble des disciplines, est confronté à ces différents problèmes et se trouve particulièrement bien placé pour organiser ces réflexions.

Le Comité d'éthique pour les sciences du CNRS sera un comité de réflexion. II devra définir parmi les nombreux problèmes posés, les priorités de sa réflexion. II devra mettre en place et nommer les membres des groupes de travail spécialisés qui auront pour mission de réfléchir, avec les laboratoires et les interlocuteurs concernés, sur les thèmes choisis. Le Comité d'éthique pour les sciences n'a pas vocation d'autorité ou de contrôle sur les laboratoires de recherche. II pourra toutefois recommander la mise en place de tels comités opérationnels auprès des instituts ou des départements scientifiques concernés.

Le Comité d'éthique pour les sciences du CNRS comprend des scientifiques de différentes disciplines et des personnalités du milieu scientifique. Les personnalités et le président sont nommés pour trois ans, renouvelables ensuite par tiers chaque année. Le Comité, sous l'autorité de son président, définit les groupes de travail et en nomme les membres.

Le Comité, une fois nommé, est indépendant de la Direction générale du CNRS mais celle-ci fera tout ce qui est en son pouvoir pour faciliter son fonctionnement et lui fournir les moyens nécessaires. En retour, le Comité devra tenir informée la Direction générale du CNRS de l'avancement de ses réflexions.

Le Comité ne doit pas être concurrentiel des autres comités d'éthique existants à l'échelon national. Ainsi, il fera rapport de l'évolution de ses réflexions au Comité consultatif national pour l'éthique dans les sciences de la vie et de la santé. Le Comité d'éthique pour les sciences se doit non seulement d'être innovant, mais également complémentaire de toute action d'envergure touchant à l'éthique des sciences, que ce soit à l'échelon national ou européen.

Le 6 juillet 1994,

Centre national de recherche scientifique

- Le domaine des sciences du comportement soulève actuellement des problèmes aigus. Les techniques de communication, d'influence, utilisant aussi bien l'hypnose que des données scientifiques psychologiques et physiologiques, envahissent la publicité. Elles montrent des frontières dangereuses chez les personnes fragiles, chez les enfants, ou aux mains des gourous des sectes. De même, avec l'extension fulgurante des outils et réseaux de communication, la création d'images virtuelles, les dérives de l'information et de la désinformation ouvrent un nouveau chapitre pour la réflexion éthique.

- Les manipulations de l'histoire existèrent de tout temps, utilisées souvent à des fins religieuses ou politiques. Or la méthodologie scientifique s'affirme, le professionnalisme des historiens permet de revoir les comportements et de réaffirmer une éthique. Ainsi le débat sur le révisionnisme peut-il devenir scientifique. Il avait dû jusqu'à présent être arbitré dans les universités françaises par un ministre (Alain Devaquet) et un maire (Michel Noir), en l'absence d'arbitrage institutionnel scientifique. Ainsi l'histoire de la France de Vichy, déformée par la politique d'après-guerre, doit-elle beaucoup sa vérité à l'Institut d'histoire du temps présent, autour de Jean-Pierre Azema et de François Bedarida. L'histoire est tant vulgarisée que l'historien est souvent dans la situation de redresser des pseudo-vérités. Récemment le centenaire de Pasteur fut ainsi l'occasion de rapprocher une image d'Épinal d'une image historique !

- Dans le domaine de l'environnement, se trace un rôle éthique très particulier des scientifiques, à la fois source d'informations inquiétantes et référence rassurante. Les scientifiques furent à l'origine de la prise de conscience des menaces sur l'ozone et l'effet de serre, mais eurent du mal à se faire entendre lors de la conférence de Rio. L'existence, à cette occasion, d'un appel d'Heidelberg de certains scientifiques craignant les excès de l'écologie, suivi du contreappel de scientifiques recadrant cette prise de position est un événement qui devrait souligner l'urgence d'un débat parmi les scientifiques. Dans ce domaine également, la responsabilité des scientifiques répandant dans la nature un agent incontrôlé nous ramène à des problèmes plus classiques rejoignant le DDT, la myxomatose en France ou l'algue Caulerpa Taxifolia en Méditerranée.

- Le problème de l'énergie nucléaire rejoint l'éthique pour les sciences à travers la responsabilité des risques, l'information sur ceux-ci, et la quasi-exclusivité des scientifiques pour assurer l'information, alors 
même que, comme dans d'autres programmes scientifiques, ils sont suspectés par le public d'appartenir à un même lobby.

NSS - Lorsque vous avez conçu ce projet de Comité d'éthique pour les sciences du CNRS, comment voyiez-vous son rôle ? Quelle position ce Comitè devait-il adopter face à des questions actuelles et préoccupantes comme celles que vous venez d'évoquer? L'objectif que vous lui assigniez était-il de rectifier certaines interprétations ou de dénoncer certains usages jugés abusifs de résultats scientifiques, d'être en somme un organisme de veille, faisant en sorte que le message scientifique ne soit pas manipulé par les médias ? S'agissait-il, autrement dit, de veiller à ce que l'image de la science transmise par les moyens de communication soit conforme à ce qu'elle est ? Ou s'agissait-il plutôt d'un travail de réflexion interne consistant à s'interroger sur la pertinence de certaines recherches compte tenu de l'utilisation qui pouvait en être faite ? Est-ce un principe de prudence appliqué à la science soulignant les risques d'une exploitation non conforme aux objectifs poursuivis dans le cas de certaines recherches, qui est à l'origine de votre démarche? Peut-on envisager d'aller jusqu'à freiner les retombées sociales de ses résultats ? Jacques Testart, quant à lui, s'est interdit la poursuite de certaines recherches. On pense actuellement que pour programmer la recherche, il n'est pas inutile de s'interroger sur le devenir et sur les retombées sociales de ses résultats.

Nous n'en sommes encore qu'à la préhistoire de l'éthique des sciences. Dans mon esprit, ce comité a pour objet de développer une conscience dans les sciences. Il aide les chercheurs à effectuer leurs travaux en toute connaissance de cause et les incite à réfléchir dans un cadre ouvert sur d'autres disciplines et sur l'environnement non scientifique. Incontestablement, les scientifiques doivent être pleinement conscients des tenants et aboutissants, des conséquences de leur recherches. Leur formation ou leur morale personnelle ne suffisent pas toujours à les y préparer.

De même, les scientifiques ont un rôle à jouer dans la diffusion des sciences. Un rôle clarificateur, une fonction d'analyste des différentes options lorsque des choix sont présentés à l'opinion publique et aux citoyens. Est-il concevable qu'un chercheur spécialiste d'une question, joue du droit prioritaire, voire exclusif, à la parole que lui confère son autorité scientifique, pour faire avaliser comme vérité ce qui n'est encore qu'une présomption, et ce, avec une absence de doute allant à l'encontre des règles de son métier et du fondement même de sa profession? Ceci revient à poser le problème de la communication entre les chercheurs et les médias, question souvent posée dans le domaine médical mais qui mérite d'être posée dans toutes les disciplines scientifiques.

NSS - C'est donc du discours des scientifiques lorsque, sortant de leur laboratoire, ils sont sollicités dans un lieu de débat public, qu'il est question. Est-ce une façon de contrôler le rôle médiatique des scientifiques ?

Il ne s'agit pas de contrôler mais de rendre le scientifique plus responsable - et pas seulement de ses résultats, mais aussi de l'utilisation médiatique qui en sera faite et de ses conséquences éventuelles sur le plan technologique. Bien sûr, il n'en porte pas la responsabilité exclusive, mais il doit en sentir le poids. L'un des effets majeurs recherchés de cette réflexion éthique est effectivement d'aider le scientifique à se sentir plus responsable.

NSS - Responsables également dans le choix de leur sujet de recherche?

Quel que soit le sujet, il est important de le mener avec responsabilité. Il est classique de mettre en cause tel biologiste ou tel chimiste, tellement passionné qu'il pousse ses expériences vers des zones dangereuses. Mais il ne faut pas considérer uniquement les dangers intrinsèques de certaines techniques. Chacun en son domaine, que ce soit l'historien, l'économiste ou le sociologue détient une responsabilité quant à sa manière de travailler. La participation à une réflexion éthique ne peut qu'accroître le sens des responsabilité des chercheurs.

NSS - Les avis et les travaux de ce comité s'adressent donc tout particulièrement aux chercheurs.

Oui, dans un premier temps du moins. Et c'est pourquoi j'ai souhaité que les conclusions et réflexions du Comité soient publics. D'après les statuts, nul n'est en état d'empêcher le Comité de publier ses remarques. 


\section{DÉCISION N 940789SJUR PORTANT CRÉATION DU COMITÉ D’ÉTHIQUE POUR LES SCIENCES - CNRS}

Le Directeur général,

Vu le décret $n^{\circ} 82-993$ du 24 novembre 1982 modifié portant organisation et fonctionnement du Centre national de la recherche scientifique :

DÉCIDE :

Art. $1^{\text {er }}$ - Un Comité d'éthique pour les sciences (COMETS) est créé au CNRS.

Instance consultative indépendante, le comité a pour mission d'émettre un avis sur les problèmes éthiques soulevés par la recherche scientifique à l'exception de ceux traités par le Comité consultatif national d'éthique pour les sciences de la vie et de la santé (CCNE).

Non concurrent des autres comités institués à l'échelon national ou européen, il contribue et développe la réflexion touchant à l'éthique des sciences.

II n'exerce aucune autorité hiérarchique sur les laboratoires de recherche.

Art. 2. - Le Comité peut être saisi par le directeur général du CNRS. II peut également se saisir de toute question qu'il juge utile d'examiner.

Ses conclusions et recommandations sont transmises au directeur général.

Art. 3. - Le président du Comité est nommé par décision du directeur général du CNRS pour une durée de 3 ans. Ce mandat peut être renouvelé.

Art. 4. - Le comité comprend, outre son président:

4-1 - quatorze membres à part entière relevant de différentes disciplines scientifiques, ou personnalités issues de la socièté civile, appartenant ou non au CNRS et qualifiés pour leur compétence et leur intérêt pour les problèmes d'éthique.

Ces membres sont nommés pour une durée de 3 ans renouvelable, par décision du directeur général du CNRS après avis des directeurs des départements scientifiques et du président du présent Comitè. Ils sont renouvelés par tiers tous les ans, le premier renouvellement ayant

NSS Et en ce qui concerne le fonctionnement futur?

Nous sommes encore dans l'utopie. Mais il me semble que ce Comité, s'il travaille bien, comme il a commencé à le faire sous lieu par tirage au sort l'année suivant l'installation du comité.

4-2 - Assistent également aux réunions à titre d'observateurs :

- un représentant du Comité consultatif national d'éthique pour les sciences de la vie et de la santé (CCNE) ;

- le président du Comité opérationnel pour l'éthique dans les sciences de la vie du CNRS (COPE) ou son représentant ;

- un représentant de l'Académie des sciences

- un représentant du CNRS ;

- les rapporteurs des divers groupes de travail définis à l'article suivant.

4-3 - Le président peut également appeler à assister aux séances toute personne dont il juge la présence utile.

Art. 5. - Dans le cadre de sa mission, le Comité définit des groupes de travail ad hoc auxquels il confie pour une durée limitée un thème de réflexion déterminé. Le président du Comité en nomme les membres.

Art. 6. - Le Comité établit un rapport annuel sur l'évolution de ses réflexions qu'il adresse à la Direction générale du CNRS.

Ce rapport est rendu public.

Le CNRS en assure la diffusion.

Art. 7. - Les séances ne sont pas publiques. Une exception à cette règle peut toutefois être décidée par le président s'il l'estime nécessaire.

Art. 8. - Le Comité peut se doter d'un règlement intérieur définissant les modalités de son fonctionnement.

Art. 9. - Le secrétariat du Comité est assuré par le CNRS sous I'autorité du président.

Art. 10. - Le support administratif et financier est assuré par la direction générale du CNRS.

La mission de l'information scientifique et technique du CNRS est chargée de la gestion de ses moyens.

Art. 11. - La présente décision sera publiée au Bulletin Officiel du Centre national de la recherche scientifique.

l'impulsion de sa présidente, est susceptible d'acquérir une grande influence. Il s'ouvrira à d'autres pays, parce que cette démarche correspond bien à l'esprit européen, comme j'ai eu l'occasion d'en discuter avec mes collègues étrangers.
Le Comité est actuellement sous la tutelle du CNRS, qui en est le moteur et le garant de son espace de liberté. Il a vocation à évoluer à terme vers une autonomie plus complète, ou plus vraisemblablement vers un accueil dans l'actuel Comité national d'éthique pour les sciences de la vie si celuici s'élargit à l'ensemble des sciences... Un morcellement en groupes de travail sera sans doute indispensable pour faire face à la multiplicité des problèmes. Mais il faudra prêter la plus grande attention à la manière dont il se fera. Mon vœu serait qu'il opère, non pas en fonction des diverses sciences, mais selon les différents problèmes. La réflexion éthique pour les sciences ne peut être qu'interdisciplinaire. Le milieu médical est déjà bien trop étroit pour traiter de bioéthique...

NSS . Rencontre-t-on dans le monde des expériences similaires ?

Les questions d'éthique sont parfois abordées au sein des institutions, le plus souvent sous un aspect opérationnel pour les sciences de la vie. La démarche choisie de la réflexion pluridisciplinaire ne pouvait guère s'engager qu'en France, pays en pointe pour l'organisation des réflexions éthiques, et à partir du CNRS, une des rares institutions omni-disciplinaire.

De plus, il existe au CNRS une tradition culturelle, un esprit critique, un constant questionnement sur la profession et les responsabilités du scientifique. L'ouverture délibérée de cette "république des savants" vers les problèmes de société a fait régner incontestablement un état d'esprit favorable à une réflexion éthique sur les sciences.

NSS - Si vous aviez à définir en quelques mots le Comité d'éthique pour les sciences du CNRS, sur quels aspects insisteriez-vous ?

Je commencerai par dire ce qu'il n'est pas. Il n'est pas l'outil de promotion d'une corporation, celle des scientifiques. Il n'est pas l'embryon d'un Conseil de l'Ordre d'une profession. Il n'est ni une instance de dissertation savante sur la complexité des problèmes ni une commission de contrôle... 


\section{COMITÉ D'ÉTHIQUE POUR LES SCIENCES \\ Liste des membres du comité à sa création}

Président :

- Hélène AHRWEILER, historienne, président de I'Université de l'Europe

Membres :

- Maurice AYMARD, historien, administrateur de la Maison des sciences de l'homme

- Georges BALANDIER, anthropologue, directeur d'études à l'EHSS

- Alain BENSOUSSAN, mathématicien, président de I'INRIA

- Hubert CURIEN, physicien, président du Conseil d'administration du CERN

- Françoise GIROUD, journaliste et écrivain

- Jacques LESOURNE, économiste, professeur au CNAM

- Jacques-Louis LIONS, mathématicien, professeur au Collège de France

- Gérard MÉGIE, physicien, directeur de I'Institut Pierre-Simon Laplace des sciences de l'environnement global

- Roger MONIER, biologiste moléculaire, directeur de recherche au CNRS, président du Conseil de la Recherche et I'Institut Gustave Roussy et du Conseil scientifique de I'Institut Curie

- Hourya SINACEUR, philosophe, président de section au Comité national d'évaluation du CNRS

Il a pour ambition de se consacrer à l'éthique de la pratique scientifique, il a pour objet de dynamiser le débat éthique parmi les scientifiques, afin d'accroître la responsabilité et le sens des responsabilités des scientifiques vis-à-vis de la société et aussi de la société visà-vis des sciences.

NSS - Comment différenciez-vous l'éthique de la morale?

Une morale, qu'on le veuille ou non, est issue de religions, de croyances globales, ses grands principes sont universels... L'éthique est avant tout pragmatique. Une éthique se construit à partir de cas concrets qui se présentent à un moment donné, dans un espace, dans un groupe social et dans un contexte culturel donnés. Ses principes durent un temps. Et une réflexion éthique, conduite à partir de cas concrets formulés par une science évolutive, pourrait bien aboutir à des
- Gérard TOULOUSE, physicien, directeur de recherche à I'ENS

- Jean-Didier VINCENT, neuroendocrinologue, professeur à I'Institut universitaire de France et à la Faculté de médecine de Paris, directeur de l'Institut Alfred Fessard du CNRS

- Michel VIVANT, juriste, professeur à I'Université de Montpellier

- Daniel WIDLOCHER, docteur en médecine et en psychologie, président du Conseil scientifique de la Faculté de médecine PitiéSalpétrière

- Dominique WOLTON, sociologue, directeur du Programme de recherche sur les sciences de la communication du CNRS

Observateurs:

- Odile FICHOT, représentant du Comité consultatif national d'éthique pour les sciences de la vie et de la santé (CCNE)

- François GROS, représentant de I'Académie des sciences

- André KASPI, représentant du CNRS

- Robert NAQUET, représentant du Comité opérationnel pour l'éthique dans les sciences de la vie au CNRS (COPE)

mixtures intéressantes, mariant diverses religions et plusieurs morales.

Né en 1934, François KOURILSKY est biologiste et médecin. II a fait une carrière de chercheur en immunologie à I'INSERM. II a fondé et dirigé plusieurs Unités de recherche et, en particulier, le Centre d'Immunologie INSERM-CNRS de Marseille. II a occupé de nombreuses fonctions de responsabilité dans la recherche, dont la présidence du Conseil de Coordination de I'Institut Curie, la viceprésidence du Conseil Supérieur de la Recherche et de la Technologie (1983-87), la présidence d'une Commission Plan Recherche (1985) et a été Directeur Général du CNRS de 1988 à 1994. II est l'auteur d'une centaine d'articles et d'ouvrages scientifiques sur les réactions immunologiques, couronnés de plusieurs prix. II est actuellement Directeur de Recherches à I'INSERM et Sous-Directeur chargé de la recherche de l'Institut Gustave Roussy. 